\title{
Massagem e Reiki para redução de estresse e melhoria de qualidade de vida: ensaio clínico randomizado
}

\author{
Massage and Reiki to reduce stress and improve quality of life: a randomized clinical trial \\ Masaje y Reiki para reducir el estrés y mejorar la calidad de vida: ensayo clínico aleatorizado
}

Como citar este artigo:

Kurebayashi LFS, Gnatta JR, Kuba G, Giaponesi ALL, Souza TPB, Turrini RNT. Massage and Reiki to reduce stress and improve quality of life: a randomized clinical trial. Rev Esc Enferm USP. 2020;54:e03612. doi: https://doi.org/10.1590/S1980-220X2018059103612

Leonice Fumiko Sato Kurebayashi, ${ }^{1,2}$

Juliana Rizzo Gnatta ${ }^{3}$

Gisele Kuba ${ }^{1,2}$

Ana Lucia Lopes Giaponesi ${ }^{1,2}$

Talita Pavarini Borges de Souza ${ }^{1,2,4}$

Ruth Natalia Teresa Turrini ${ }^{3}$

${ }^{1}$ Universidade de São Paulo, Escola de Enfermagem, Programa de

Pós-Graduação em Enfermagem na Saúde do Adulto, São Paulo, SP, Brasil.

${ }^{2}$ Instituto de Terapia Integrada e Oriental, Curso de Massoterapia, São Paulo, SP, Brasil.

${ }^{3}$ Universidade de São Paulo, Escola de Enfermagem, Departamento de Enfermagem Médico-Cirúrgica, São Paulo, SP, Brasil.

${ }^{4}$ Faculdade de Ciências Médicas da Santa Casa de São Paulo, São Paulo, SP, Brasil.

\section{ABSTRACT}

Objective: To verify if a Massage (Anma technique) followed by rest or Reiki helps to reduce stress levels and improve the quality of life of individuals seen in an outpatient clinic of integrative practices. Method: A randomized controlled clinical trial conducted with 122 individuals randomized into three groups: G1 - Massage followed by rest; G2 - Massage followed by Reiki; and G3 - control (no intervention). Stress and Quality of Life levels were measured using the LSS and SF-12v2 instruments, which were applied before and after the intervention. Results: The study was conducted with 101 participants. Massages followed by rest (G1) or Reiki (G2) proved to be effective in reducing stress levels and improving quality of life when compared to the Control Group (G3). The best results obtained by the group which received Massage followed by Reiki (G2) were observed in the mental domain of quality of life, emphasizing the scope of Reiki effects on mental and psychological aspects. Conclusion: Massage followed by rest and Massage followed by Reiki application were effective in reducing stress levels and improving quality of life. Brazilian Registry of Clinical Trials: RBR-42c8wp.

\section{DESCRIPTORS}

Massage; Complementary Therapies; Stress, Psychological; Quality of Life; Holistic Nursing.

Autor correspondente:

Leonice Fumiko Sato Kurebayashi

Rua Vieira Fazenda, 80, Vila Mariana CEP 04117-030 - São Paulo, SP, Brasil fumieibez@gmail.com 


\section{INTRODUÇÃO}

A palavra estresse tem sido associada a sensações de desconforto, e o número de pessoas que se definem como estressadas é crescente. Um elemento estressor pode ser uma situação ou experiência que gera um desafio e estimula uma resposta por parte do indivíduo ${ }^{(1)}$. Geralmente o estresse é concebido como algo negativo, que prejudica o desempenho global do indivíduo; no entanto, o estressor pode ser enfrentado de modo positivo e construtivo a depender de como $o$ indivíduo reage em relação ao desafio. Tanto a adaptação quanto o enfrentamento são processos individuais que requerem equilíbrio entre habilidades, capacidades, apoio social e os desafios e estressores encontrados ${ }^{(2)}$.

$\mathrm{O}$ estresse configura-se como um fenômeno psiconeuroimunológico complexo, que afeta o indivíduo física, mental, emocional e socialmente. Do ponto de vista endócrino e neurológico, durante o enfrentamento do estresse, o indivíduo passa por uma adaptação, denominada Síndrome de Adaptação Geral, composta por três fases. $\mathrm{Na}$ fase de alerta, gera-se uma reação de luta ou fuga com alterações fisiológicas como o aumento do débito cardíaco, da frequência respiratória e do catabolismo ${ }^{(3)}$. Se o estressor perdurar, o indivíduo entrará na Fase de Resistência (período de adaptação), em que ocorrerá o consumo de reservas energéticas adaptativas em busca do reequilíbrio, alteração nas suprarrenais caracterizada por diminuição na produção de adrenalina pela medula e aumento de corticosteroides pelo córtex adrenal ${ }^{(3)}$. Emocionalmente, o indivíduo poderá experimentar sentimentos de medo, frustração, conflitos, ansiedade, depressão, mal-estar, apatia, alienação, hipocondria, e uma série de alterações bioquímicas afetarão diretamente as condições imunológicas do indivíduo ${ }^{(3)} \cdot \mathrm{Na}$ persistência do estressor, as reservas de energia adaptativa acabarão e a pessoa entrará na terceira fase, a de Exaustão ${ }^{(3)}$. A falha da resposta adaptativa pode desencadear o desenvolvimento de inúmeras doenças, prejudicar a qualidade de vida $(\mathrm{QV})$ e a produtividade do indivíduo. A QV está intrinsecamente relacionada aos níveis de estresse, tanto em relação ao estado de saúde quanto às áreas social, afetiva e profissional ${ }^{(4)}$.

Quanto às diferentes abordagens para lidar com estresse, promoção de bem-estar e melhoria de $\mathrm{QV}$, há um crescente interesse sobre a utilização das Práticas Integrativas e Complementares (PIC), como a Massagem e o Reiki.

No Brasil, a Política Nacional de Práticas Integrativas e Complementares em Saúde datada de 2006, contemplava cinco diretrizes: medicina tradicional chinesa, medicina antroposófica, homeopatia, uso de plantas medicinais/fitoterapia e o uso de águas termais ${ }^{(5)}$. A Portaria n 849 de 2017 incluiu mais 14 práticas à Política Nacional, dentre elas o Reiki ${ }^{(6)}$, e no ano seguinte, foram acrescentadas mais 10 , representando assim, um total de 29 PIC regulamentadas a serem realizadas pelos profissionais de saúde ${ }^{(7)}$.

O Conselho Federal de Enfermagem (COFEN), por meio da Resolução n ${ }^{\circ}$ 581/2018, determinou os procedimentos para se registrar títulos de pós-graduação concedidos e aprovou a lista das especialidades dos profissionais, de modo que a "Enfermagem em Práticas Integrativas e Complementares" está inserida na Área I - Saúde Coletiva, Saúde da Criança e do Adolescente, Saúde do Adulto ${ }^{(8)}$. A publicação da Resolução 585/2018, que versa especificamente sobre o reconhecimento da acupuntura como uma especialidade e/ou qualificação do profissional de Enfermagem $^{(9)}$, reforça a presença dessa profissão na atuação das PIC no contexto do cuidar.

Há Teorias de Enfermagem que embasam conceitualmente a aplicação das PIC na implementação do cuidado. Martha Rogers, em sua visão holística, definiu modalidades não invasivas como centrais para a assistência de enfermagem no sentido de trazer bem-estar e $\mathrm{QV}^{(10)}$. Nesse sentido, a Massagem e o Reiki são modalidades de PIC apropriadas para a prática assistencial. Um estudo mostrou que uma única sessão com Massagem ou Reiki promoveram melhoras clínicas similares em pacientes hospitalizados com náusea, dor, fadiga, ansiedade, depressão e bem-estar, sendo que o Reiki apresentou resultados superiores à Massagem em relação à fadiga e ansiedade ${ }^{(11)}$.

No presente estudo aplicou-se a Massagem relaxante Anmá, de origem japonesa, que melhora ou alivia sintomas físicos e psicológicos ${ }^{(12)}$. A Massagem terapêutica é um método não farmacológico recomendável como prática de Enfermagem para pacientes críticos em terapia intensiva que auxiliou na redução de níveis de cortisol em pacientes coronariopatas estressados ${ }^{(13)}$.

O Reiki, uma técnica terapêutica energética complementar e adjuvante, pode reduzir o estresse e promover relaxamento, inclusive por meio do autocuidado, pelo fato de também ser autoaplicável ${ }^{(14)}$. Estudo de revisão identificou o Reiki como uma técnica efetiva para a redução da dor ${ }^{(15)}$, há relatos de alívio da dor crônica em idosos ${ }^{(16)}$ e melhoria de $\mathrm{QV}$, humor e redução de efeitos colaterais de quimioterapia $^{(17)}$. Estudo realizado com idosos verificou que o Reiki produziu efeitos psicofisiológicos e melhoria da $\mathrm{QV}$ em indivíduos que apresentavam sintomas de estresse ${ }^{(18)}$. Embora esta técnica seja utilizada para uma variedade de sintomas, tanto físicos quanto psicológicos, publicações que demonstrem sua efetividade na prática assistencial de enfermagem ainda são poucas ${ }^{(19)}$, e mais estudos utilizando-se a técnica do Reiki são necessários.

Em relação à Massagem, seu uso na prática assistencial de enfermagem à pessoa idosa no domicílio contribuiu para melhorar a saúde e bem-estar e influenciou positivamente fatores como dor, sono, estado emocional e saúde psicossocial $^{(20)}$. Também relataram-se efeitos de relaxamento sobre os músculos mastigatórios, com redução de dor em indivíduos com bruxismo e melhoria de $\mathrm{QV}^{(21)}$.

Devido à importância de se discutir formas de enfrentamento para o estresse que contribuam para melhoria da $\mathrm{QV}$, o presente estudo teve como objetivo verificar se a Massagem (técnica Anmá) seguida de repouso ou Reiki, poderia auxiliar na redução dos níveis de estresse e na melhoria da QV de indivíduos atendidos em ambulatórios de PIC na cidade de São Paulo. A hipótese do estudo foi "a Massagem seguida de Reiki é mais efetiva do que a Massagem seguida de repouso". 


\section{MÉTODO}

\section{Desenho do estudo}

Estudo prospectivo, simples cego (cegamento estatístico), do tipo ensaio clínico controlado randomizado, com três grupos: Grupo Massagem seguida de repouso (G1), Grupo Massagem seguida de Reiki (G2) e Grupo Controle sem tratamento (G3).

\section{População}

Constituída por voluntários que buscaram atendimento em um ambulatório de PIC e por militares do IV-Comando Aéreo Regional de São Paulo (IV-COMAR) no segundo semestre de 2015.

\section{CRITÉrIOS DE SELEÇÃo}

Foram incluídos os participantes que apresentaram pontuação entre 37 a 119 pela Lista de Sintomas de Stress de Vasconcellos (LSS) ${ }^{(22)}$. Foram excluídos: gestantes, participantes com férias previstas ou em licença médica no período da pesquisa, em uso de ansiolíticos e antidepressivos, com relato de desconforto durante a Massagem ou com lesão tecidual em pontos a serem massageados.

Dos 141 interessados em participar do estudo, 122 atenderam aos critérios de seleção e foram randomizados por meio do programa Research Randomizer Quick Tutorial. O número mínimo de 30 participantes por grupo foi determinado por dimensionamento amostral prévio com poder de $80 \%$ e nível de confiança de $95 \%$.

Para o G1 foi aplicado um protocolo de Massagem Anmá por 20 minutos, seguido de repouso de $10 \mathrm{minu}-$ tos. O G2 recebeu o protocolo de Massagem Anmá por 20 minutos e em seguida o de Reiki por 10 minutos e $\mathrm{G} 3$ não recebeu qualquer protocolo de intervenção.

\section{INTERVENÇÕES}

A Massagem Anmá foi realizada na região posterior cervical, torácica, lombar, glúteo e coxas até os pés, com duração de 20 minutos e incluiu as técnicas de alisamento, pressão e amassamento. $\mathrm{O}$ alisamento pode ser definido como a realização de movimento contínuo com leve pressão; a pressão foi realizada por meio da compressão do ponto anatômico por aproximadamente um segundo; o amassamento foi realizado com três movimentos circulares durante a pressão. O protocolo de aplicação da técnica da Massagem foi realizado na seguinte sequência: deslizamento bilateral com a palma das duas mãos sobre a coluna (2 vezes); pressão e amassamento sobre paravertebrais (2 vezes); amassamento desde a raiz da espinha da escápula até ângulo superior da escápula (2 vezes); pressão e amassamento sobre a porção média de trapézio (2 vezes); pressão sobre a linha occipital ( 2 vezes); amassamento sobre a cervical (3 vezes); pressão e amassamento sobre o glúteo - 2 linhas ( 2 vezes); pressão sobre face posterior da coxa - 2 linhas (2 vezes); pressão sobre linha poplítea - (2 vezes); amassamento com região supracarpiana sobre tríceps sural - (2 vezes); pressão e amassamento sobre região plantar dos pés- 3 linhas (2 vezes).
O protocolo de Reiki considerou o posicionamento das mãos espalmadas próxima aos olhos, região occipital, região laríngea e sobre o esterno ou região cardíaca, durante 2,5 minutos em cada região, totalizando 10 minutos de aplicação da técnica.

As intervenções foram realizadas duas vezes por semana, totalizando oito sessões em um mês de atendimento. Para garantir a uniformidade dos tratamentos, uma equipe de 11 pessoas foi treinada pelos próprios profissionais do ambulatório de PIC em que foi realizada a coleta de dados. A equipe foi constituída por estudantes do Curso Técnico de Massagem e monitorados por professores experientes nas técnicas.

\section{Coleta de Dados}

Os dados foram coletados por meio da aplicação de dois instrumentos: a Lista de Sintomas de Stress de Vasconcellos, (LSS) que avalia os níveis de estresse do indivíduo ${ }^{(22)}$ e o $\mathrm{SF}-12 \mathrm{v} 2^{(23)}$ para a QV. Os instrumentos foram aplicados em dois momentos: antes da intervenção $(\mathrm{t} 1)$ e ao término da mesma ( 2 2).

A LSS é composta por 59 sintomas psicofisiológicos e psicossociais de estresse para os quais os entrevistados devem assinalar a presença e a frequência de cada sintoma ( 0 a 3), perfazendo um total de 177 pontos. Os escores mais baixos indicam ausência ou baixa frequência de manifestação de sintomas e os escores mais altos, o inverso ${ }^{(22)}$.

O SF-12v2 é uma versão mais curta do instrumento SF36 e utiliza 12 perguntas para medir a saúde funcional e bem-estar do ponto de vista do paciente, abrangendo os mesmos oito domínios de saúde que o SF-36v2 ${ }^{(23)}$. Optou-se por utilizar a segunda versão do SF12 no presente estudo porque este instrumento teve suas propriedades psicométricas validadas para a língua portuguesa e os resultados mostraram evidências confiáveis ao seu uso como medida de saúde física e saúde mental no contexto brasileiro ${ }^{(24)}$.

\section{ANÁlISE E TRATAMENTO DOS DADOS}

Os dados foram descritos por frequências relativas e medidas de tendência central. A comparação entre os grupos foi feita pelo teste do qui-quadrado de Pearson para as variáveis qualitativas, ANOVA para testar as diferenças de média e teste de Levene para verificar a igualdade de variância. Para comparar o antes e depois foi utilizado o teste ANOVA para medidas repetidas e o teste de Tukey para as comparações múltiplas. Adotou-se nível de significância a $=5 \%$ e o tamanho do efeito das intervenções foi medido pelo teste $\mathrm{d}$ de Cohen, o qual determina o índice: $\geq-0,15 \mathrm{e}<0,15$ (efeito insignificante); $\geq 0,15 \mathrm{e}<0,40$ (pequeno efeito); $\geq 0,40$ e $<0,75$ (médio efeito); $\geq 0,75$ e $<1,10$ (grande efeito); $\geq 1,10<1,45$ (muito grande efeito); > 1,45 (enorme efeito). Para os percentuais de mudanças e suas classificações: < 75 (redução enorme); $\leq-50$ e $>-75$ (redução muito grande); $\leq-30$ e $>-50$ (redução grande); $\leq-15$ e $>-30$ (redução média); $\geq-5$ e $<5$ (mudança insignificante); $\geq 5$ e $<15$ (aumento pequeno); $\geq 15$ e $<30$ (aumento médio); $\geq 30$ e $<50$ (aumento grande) $\geq 50$ e $<75$ (aumento muito grande); $>75$ (aumento enorme). 


\section{AsPeCTOS ÉTIICOS}

O estudo foi aprovado pelo Comitê de Ética em Pesquisa da Escola de Enfermagem da Universidade de São Paulo, sob o Parecer no 1.105.429/2015. Todos os participantes preencheram o Termo de Consentimento Livre e Esclarecido. O número de registro do ensaio pelo Registro Brasileiro de Ensaios Clínicos foi RBR-42c8wp.
Não foram observados prejuízos ou danos aos participantes decorrentes do estudo.

\section{RESULTADOS}

Finalizou-se o estudo com 101 participantes. Vinte e uma pessoas não concluíram o protocolo pelos seguintes motivos: perda da continuidade dos tratamentos (13) e questionários não respondidos (8), como observado na Figura 1.

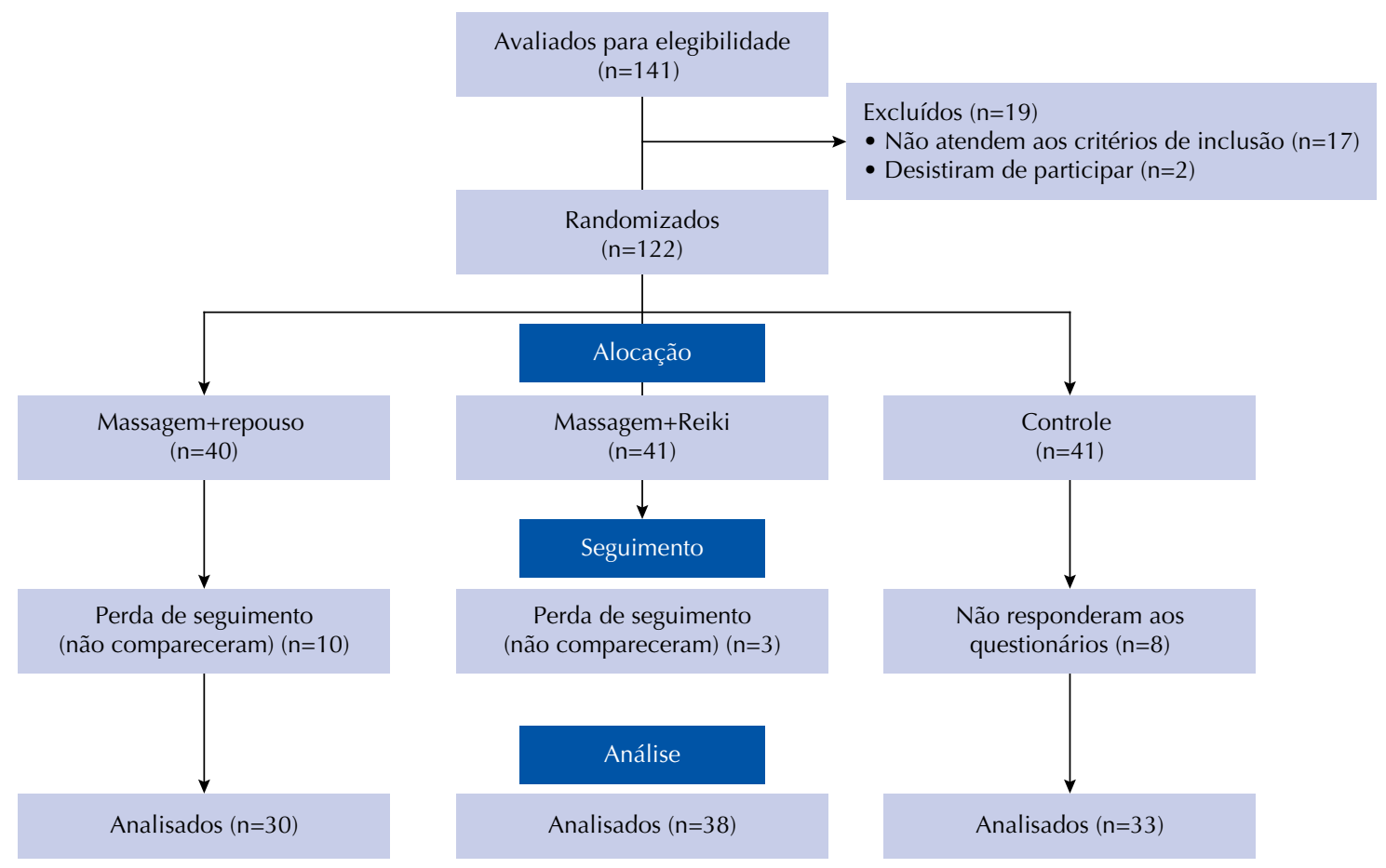

Figura 1 - Fluxograma de participantes envolvidos no estudo.

Houve maior participação do sexo feminino (66,0\%), com distribuição homogênea intergrupos $(p=0,738)$ e com relação à categoria dos profissionais, 29,7\% (30) eram militares, 14,9\% (15) profissionais da saúde, 8,9\% (9) estudantes e $46,5 \%$ (47) outros. A idade média dos participantes entre os grupos oscilou em torno de 35 anos, os grupos se mostraram homogêneos, no momento inicial da intervenção (t1), em relação à idade $(\mathrm{p}=0,450)$, ao escore dos instrumentos LSS $(\mathrm{p}=0,865), \mathrm{SF} 12 \mathrm{v} 2$-físico $(\mathrm{p}=0,668)$ e SF12v2-mental $(\mathrm{p}=0,954)$.

$\mathrm{Na}$ análise comparativa dos grupos, houve diferença estatística significativa entre o antes e após a intervenção para os níveis de estresse e domínios físico e mental de QV (Tabela 1).

Tabela 1 - Comparação de medidas de tendência central antes e após intervenção dos níveis de estresse (LSS) e qualidade de vida domínios físico e mental (SF12v2) de acordo com os grupos de estudo - São Paulo, SP, Brasil, 2015.

\begin{tabular}{|c|c|c|c|c|}
\hline & G1 & G2 & G3 & \\
\hline Escalas & Média (DP) & Média (DP) & Média (DP) & $\mathrm{p}^{*}$ \\
\hline $\mathrm{LSS}_{\mathrm{t1}}$ & $66,3(20,6)$ & $67,7(23,4)$ & $69,2(19,7)$ & \\
\hline $\mathrm{LSS}_{\mathrm{t} 2}$ & $50,4(21,0)$ & $45,5(22,8)$ & $65,9(20,1)$ & $<0,001$ \\
\hline SF12v2-físico & $48,4(7,1)$ & $47,3(7,8)$ & $46,6(9,3)$ & \\
\hline SF12v2-físico & $51,7(5,8)$ & $50,1(6,1)$ & $45,8(8,4)$ & 0,034 \\
\hline SF12v2-mental ${ }_{t 1}$ & $37,8(8,9)$ & $37,2(10,0)$ & $37,1(10,6)$ & \\
\hline SF12v2-mental ${ }_{\mathrm{t} 2}$ & $44,5(8,1)$ & $47,4(10,5)$ & $37,2(11,0)$ & 0,001 \\
\hline
\end{tabular}

* ANOVA de medidas repetidas. 
O teste de Tukey mostrou que as diferenças ocorreram entre os Grupos G2 e G3 no escore do LSS e do SF12v2-mental, e entre os Grupos G1 e G3 no SF12v2-físico.
Pela análise intragrupo do tamanho de efeito pelo d de Cohen, observou-se que houve redução dos níveis de estresse apenas para G1 e G2. Em relação a melhoria de qualidade de vida, houve melhora dos escores do domínio físico e mental para os dois grupos de intervenção (Tabela 2).

Tabela 2 - Tamanho do efeito (Cohen's D) e percentual de mudança da intervenção intragrupo medida pelo LSS e instrumento de avaliação de qualidade de vida nos três grupos de estudo - São Paulo, SP, Brasil, 2015.

\begin{tabular}{lccccccc}
\hline Grupos & N & Cohen's d & $\%$ & Cohen's d & $\%$ & Cohen's d & $\%$ \\
\hline & & LSS & & SF12v2-físico & & SF12v2-mental \\
G1 & 30 & 0,78 & $-24,0$ & 0,52 & $+7,0$ & 0,8 & $+18,0$ \\
G2 & 38 & 0,98 & $-33,0$ & 0,40 & $+6,0$ & 1,0 & $+27,0$ \\
G3 & 33 & 0,17 & $-5,0$ & 0,09 & $-2,0$ & 0,01 & 0,0 \\
\hline
\end{tabular}

Observou-se que as intervenções em G1 e G2 apresentaram tamanho de efeito grande pelo d Cohen, com média e elevada redução dos escores de estresse, respectivamente. Para a medida QV, as intervenções em G1 e G2 tiveram tamanho de efeito médio e produziram pequena melhora no domínio físico. Em relação ao domínio mental, para esses dois grupos as intervenções foram de grande efeito e a melhora da QV foi média.

\section{DISCUSSÃO}

No presente estudo, os dois grupos de intervenção utilizaram a Massagem e ambos, evidenciaram diferenças significativas após o tratamento em relação ao controle. Contudo, os resultados da presente investigação mostraram que a Massagem seguida pela aplicação do Reiki (G2) conseguiu melhor efeito sobre os escores de QV no domínio mental do que o grupo Massagem seguida de repouso (G1).

De fato, o Reiki tem sido utilizado para reduzir problemas de natureza psicológica. O Reiki conseguiu diminuir os escores médios de dor, inclusive as dores crônicas em idosos de forma significativa ${ }^{(14,16)}$, bem como redução da ansiedade, frequência cardíaca e respiratória de crianças em cuidados paliativos ${ }^{(25)}$. Uma vantagem importante desta técnica é de ser autoaplicável, sendo utilizada com sucesso em estudantes universitários acompanhados por 20 semanas para redução do estresse e maior relaxamento ${ }^{(14)}$.

O Reiki parece ter sido decisivo para ampliar os resultados positivos nos níveis de estresse no presente estudo. $\mathrm{Na}$ tentativa de buscar entender tais resultados, reconhece-se que pouco se sabe sobre os mecanismos de ação desta prática e ressalta-se a necessidade de agregar conhecimentos multidisciplinares, como o da física quântica.

A linguagem da física quântica tem sido apropriada para dar conta da noção de energia vital, tanto na espiritualidade como nas PIC. A mesma tem sido explicada a partir da ideia dos estímulos aos neurotransmissores ou dos impulsos bioelétricos que transportam informações pelo corpo. Desse modo, a energia vital seria uma informação organizacional que direcionaria as estruturas subatômicas e poderia ser modulada pelo pensamento, dado que este também é composto por ondas vibracionais. Assim, a intenção do terapeuta para a cura seria muito importante, por transmitir essa informação para o corpo sutil do paciente ${ }^{(26)}$.

A partir do entendimento de que os seres humanos são campos de energia, é possível especular que o campo é afetado por uma intenção de cuidado, ou pela energia sutil sensível às mãos de praticantes de terapias como o Reiki. O campo energético que se forma no encontro entre duas pessoas é resultante da interação entre a consciência de ambos; e no cuidado prestado a alguém, destaca-se o papel desta consciência, por meio da atenção e da intencionalidade na direção da saúde e do bem-estar ${ }^{(26)}$.

Por outro lado, a Massagem em suas diferentes modalidades técnicas tem sido investigada há mais tempo, com resultados satisfatórios: na gestão de sintomas como dor, náusea, ansiedade, tensão, fadiga e depressão em pacientes hospitalizados ${ }^{(11,26)}$; diminuição da lombalgia ocupacional, com melhora nas atividades de trabalho da equipe de Enfermagem após aplicação de Massagem por acupressão ${ }^{(27)}$; melhora de parâmetros fisiológicos do estresse, diminuição da ansiedade, tensão muscular, dor e flexibilidade em pacientes com dor nas costas pelo uso da Massagem tailandesa $^{(28)}$. Embora diversos estudos explicitem a eficácia da Massagem, esta prática não foi incluída na última publicação do Ministério da Saúde brasileiro a respeito das práticas que integram a Política Nacional de $\mathrm{PIC}^{(7)}$.

Como limitação do presente estudo, cita-se a ausência de grupo placebo para o Reiki, não sendo possível, portanto, avaliar o efeito da técnica isoladamente na redução do estresse e melhoria de QV. A fim de que possam ser aprofundados os debates sobre aspectos concernentes ao Reiki, propõem-se desenhos que apresentem grupo placebo, permitindo avaliar o efeito desta prática isoladamente, conforme proposto em pesquisa que mostrou resultados positivos na melhoria da QV de idosos com sintomas de estresse que receberam Reiki ${ }^{(18)}$.

Por fim, considera-se que as PIC podem ter um papel relevante na Enfermagem para a construção de práticas assistenciais voltadas à integralidade. A Teoria de Rogers subsidia que as práticas assistenciais da profissão sejam 
embasadas em um forte aspecto holístico ${ }^{(10)}$. Nesse sentido, este estudo contribui para a disseminação de terapias como Massagem e Reiki que podem beneficiar o indivíduo por meio da redução dos níveis de estresse e promover a melhoria da $\mathrm{QV}$, prevenindo-o de enfermidades futuras. Além disso, estudos sobre tais práticas reforçam a atuação do papel do enfermeiro como pesquisador nessa área, contribuindo cientificamente para que as PIC sejam incluídas no ensino e na extensão de cursos formadores de profissionais da saúde.

Trabalhos como este, que explicitam o tempo, o tipo, e a forma de aplicação das práticas complementares em questão, contribuem para agregar conhecimento à área das PIC e da Enfermagem, inclusive embasado pelo referencial teórico desta profissão. A Enfermagem, fundamentada em princípios humanistas e holísticos associados ao relacionamento interpessoal, é campo vasto a ser explorado pelas PIC, inclusive por meio da inserção dessas práticas nos cursos de graduação da área da saúde. O profissional que teve contato com diferentes práticas de cuidado durante sua formação será capaz de aprimorar-se e implementar esse conhecimento no exercício da prática assistencial. As PIC aplicadas juntamente aos planos terapêuticos tradicionais de promoção à saúde, resultará em uma abordagem que visa o bem-estar físico, mental e emocional dos pacientes.

\section{CONCLUSÃO}

A Massagem seguida de repouso e a Massagem seguida pela aplicação de Reiki se mostraram efetivas na redução dos níveis de estresse e melhoria de qualidade de vida, com resultados superiores para o Grupo Massagem seguida de Reiki quando comparada ao Grupo Controle. Em relação à $\mathrm{QV}$, melhores resultados foram encontrados para o domínio mental, enfatizando a abrangência dos efeitos do Reiki sobre os aspectos psicoemocionais. Sugere-se a realização de estudos que contemplem grupo placebo para Reiki, a fim de avaliar o alcance dessa prática isoladamente e associada à Massagem, bem como associar a medição de parâmetros fisiológicos para verificar o efeito das técnicas sobre o estresse e, consequentemente, na melhoria da QV.

\section{RESUMO}

Objetivo: Verificar se a Massagem (técnica Anmá) seguida de repouso ou Reiki auxilia na redução dos níveis de estresse e na melhoria da qualidade de vida de indivíduos atendidos em um ambulatório de práticas integrativas. Método: Ensaio clínico controlado randomizado conduzido com 122 indivíduos randomizados em três grupos: G1 - Massagem seguida de repouso, G2 - Massagem seguida de Reiki e G3 - controle (sem intervenção). Os níveis de estresse e Qualidade de Vida foram mensurados por meio dos instrumentos LSS e SF-12v2, os quais foram aplicados antes e após intervenção. Resultados: Finalizou-se o estudo com 101 participantes. As Massagens seguidas de repouso (G1) ou de Reiki (G2) se mostraram efetivas na redução dos níveis de estresse e melhoria de qualidade de vida quando comparadas ao Grupo Controle (G3). Os melhores resultados obtidos pelo grupo que recebeu Massagem seguida de Reiki (G2) foram observados no domínio mental de qualidade de vida, enfatizando a abrangência dos efeitos do Reiki sobre os aspectos mentais e psicológicos. Conclusão: A Massagem seguida de repouso e a Massagem seguida pela aplicação de Reiki se mostraram efetivas na redução dos níveis de estresse e melhoria de qualidade de vida. Registro Brasileiro de Ensaios Clínicos: RBR-42c8wp.

\section{DESCRITORES}

Massagem; Terapias Complementares; Estresse Psicológico; Qualidade de Vida; Enfermagem Holística.

\section{RESUMEN}

Objetivo: Verificar si el Masaje (técnica ANMA) seguido de reposo o Reiki auxilia en la reducción de los niveles de estrés y en la mejora de la cualidad de vida de individuos atendidos en un ambulatorio de prácticas integrativas. Método: Ensayo clínico controlado aleatorizado conducido con 122 individuos aleatorizado en tres grupos: G1 - Masaje seguida de reposo, G2 - Masaje seguida de Reiki y G3 - control (sin intervención). Los niveles de estrés y Cualidad de Vida fueron mensurados través de los instrumentos LSS y SF-12v2, para los cuales fue aplicada intervención dantes y después. Resultados: Finalizó se el estudio con 101 participantes. Las Masajes seguidas de reposo (G1) o de Reiki (G2) se mostrarán efectivas en la reducción de los niveles del estrés y mejora de la cualidad de vida cuando comparadas al Grupo Control (G3). Los mejores resultados obtenidos por el grupo que recibió Masaje seguida de Reiki (G2) fueran observados en los dominios mentales de cualidad de vida, enfatizando el alcance de los efectos del Reiki sobre los aspectos mentales y psicológicos. Conclusión: La Masaje seguida del reposo y la Masaje seguida de la aplicación del Reiki mostraran se efectivas en la reducción de los niveles de estrés y mejora de la cualidad de vida. Registro Brasileño de Ensayos Clínicos: RBR-42c8wp.

\section{DESCRIPTORES}

Masaje; Terapias Complementarias; Estrés Psicológico; Calidad de Vida; Enfermería Holística.

\section{REFERÊNCIAS}

1. Sousa MBC, Silva HPA, Galvão-Coelho NL. Resposta ao estresse: I. Homeastase e teoria da alostase. Est Psic (Natal). 2015;20(1):2-11. DOI: 10.5935/1678-4669.20150002

2. Benetti ERR, Stumm EMF, Weiller TH, Batista KM, Lopes LFD, Guido LA. Coping strategies and characteristics of the nursing staff of a private hospital. Rev Rene. 2015;16(1):3-10. DOI: 10.15253/2175-6783.2015000100002

3. Selye H. Stress and the general adaptation syndrome. Br Med J. 1950;1(4667):1383-92.

4. Kurebayashi LFS, Turrini RNT, Kuba G, Shimizu MHM, Takiguchi RS. Chinese phytotherapy to reduce stress, anxiety and improve quality of life: randomized controlled trial. Rev Esc Enferm USP. 2016;50(5):853-60. DOI: 10.1590/S0080-623420160000600020

5. Brasil. Ministério da Saúde. Política Nacional de Práticas Integrativas e Complementares no SUS: atitude de ampliação de acesso. Brasília: MS; 2006. 
6. Brasil. Ministério da Saúde. Portaria n 849/2017. Inclui a Arteterapia, Ayurveda, Biodança, Dança Circular, Meditação, Musicoterapia, Naturopatia, Osteopatia, Quiropraxia, Reflexoterapia, Reiki, Shantala, Terapia Comunitária Integrativa e Yoga à Política Nacional de Práticas Integrativas e Complementares (PNPIC) no Sistema Único de Saúde [Internet]. Brasília; 2017 [citado 2019 abr. 19]. Disponível em: http://bvsms.saude.gov.br/bvs/saudelegis/gm/2017/prt0849_28_03_2017.html

7. Brasil. Ministério da Saúde. Portaria n. 702/2018. Altera a Portaria de Consolidação n $2 / G M / M S$, de 28 de setembro de 2017, para incluir novas práticas na Política Nacional de Práticas Integrativas e Complementares - PNPIC [Internet]. Brasília; 2018 [citado 2019 abr. 19 ]. Disponível em: http://bvsms.saude.gov.br/bvs/saudelegis/gm/2018/prt0702_22_03_2018.html

8. Conselho Federal de Enfermagem (COFEN). Resolução COFEN n· 581/2018. Atualiza, no âmbito do Sistema COFEN/Conselhos Regionais de Enfermagem, os procedimentos para Registro de Títulos de Pós-Graduação Lato e Stricto Sensu concedido a Enfermeiros e aprova a lista das especialidades [Internet]. Brasília; 2018 [citado 2019 abr. 19]. Disponível em: http://www.cofen.gov.br/resolucao-cofenno-581-2018_64383.html

9. Conselho Federal de Enfermagem (COFEN). Resolução COFEN no 585/2018. Estabelece e reconhece, ad referendum do Plenário do Conselho Federal de Enfermagem, a Acupuntura como especialidade ou qualificação do profissional Enfermeiro(a) [Internet]. Brasília; 2018 [citado 2019 abr. 19]. Disponível em: http://www.cofen.gov.br/resolucao-cofen-no-585-2018_64784.html

10. McEwen M, Wills EM. Bases teóricas para enfermagem. 4ª ed. Porto Alegre: Artmed; 2016.

11. Vergo MT, Pinkson BM, Broglio K, Li Z, Tosteson TD. Immediate symptom relief after a first session of massage therapy or Reiki in hospitalized patients: a 5-year clinical experience from a rural academic medical center. J Altern Complement Med. 2018;24(8):801-8. DOI: 10.1089/acm.2017.0409

12. Donoyama N, Satoh T, Hamano T. Effects of Anma massage therapy (Japanese massage) for gynecological cancer survivors: study protocol for a randomized controlled trial. Trials. 2013;14:233. DOI: 10.1186/1745-6215-14-233

13. Adib-Hajbaghery M, Rajabi-Beheshtabad R, Arjmand A. Comparing the effect of whole body massage by a specialist nurse and patients relatives on blood cortisol level in coronary patients. ARYA Atheroscler. 2015;11(2):126-32.

14. Bukowski EL. The use of self-Reiki for stress reduction and relaxation. J Integr Med. 2015;13(5):336-40. DOI: 10.1016/S20954964(15)60190-X

15. Demir Doğan M. The effect of Reiki on pain: a meta-analysis. Complement Ther Clin Pract. 2018;31:384-7. DOI: 10.1016/j.ctcp.2018.02.020

16. Freitag VL, Dalmolin IS, Badke MR, Andrade A. Benefits of Reiki in older individuals with chronic pain. Texto Contexto Enferm. 2014;23(4):1032-40. DOI: 10.1590/0104-07072014001850013

17. Orsak G, Stevens AM, Brufsky A, Kajumba M, Dougall AL. The effects of Reiki therapy and companionship on quality of life, mood, and symptom distress during chemotherapy. J Evid Based Complementary Altern Med. 2015;20(1):20-7. DOI: 10.1177/2156587214556313

18. Oliveira RMJ. Efeitos da prática do Reiki sobre aspectos psicofisiológicos e de qualidade de vida de idosos com sintomas de estresse: estudo placebo e randomizado [tese doutorado]. São Paulo: Universidade Federal de São Paulo, Escola Paulista de Medicina; 2013.

19. Freitag VL, Andrade A, Badke MR. The Reiki as therapeutic form in health care: a narrative literature review. Enfermería Global. 2015;14(2):335-6. DOI: 10.6018/eglobal.14.2.200511

20. Mcfeeters S, Pront L, Cuthbertson L, King L. Massage, a complementary therapy effectively promoting the health and well-being of older people in residential care settings: a review of the literature. Int J Older People Nurs. 2016;11(4):266-83. DOI: 10.1111/opn.12115

21. Gomes CAFP, El-Hage Y, Amaral AP, Herpich CM, Politti F, Kalil-Bussadori S, et al. Effects of massage therapy and occlusal splint usage on quality of life and pain in individuals with sleep bruxism: a randomized controlled trial. J Jpn Phys Ther Assoc. 2015;18(1):1-6. DOI: 10.1298/jjpta.Vol18_001

22. Ferreira EAG, Marques AP, Matsutani LA, Vasconcelos EG, Mendonça LLF. Avaliação da dor e estresse em pacientes com fibromialgia. Rev Bras Reumatol. 2002;42(2):104-10.

23. Utah Health Status Survey. Interpreting the SF-12. Salt Lake City: Utah Department of Health; 2001.

24. Damásio BF, Andrade TF, Koller SH. Psychometric properties of the Brazilian 12-Item Short-Form Health Survey Version 2 (SF-12v2). Paideia (Ribeirão Preto). 2015;25(60):29-37. DOI: 10.1590/1982-43272560201505

25. Thrane SE, Maurer SH, Ren D, Danford CA, Cohen SM. Reiki therapy for symptom management in children receiving palliative care: a pilot study. Am J Hosp Palliat Care. 2016;34(4):373-9. DOI: 10.1177/1049909116630973

26. Rodgers NJ, Cutshall SM, Dion LJ, Dreyer NE, Hauschulz JL, Ristau CR, et al. A decade of building massage therapy services at an academic medical center as part of a healing enhancement program. Complement Ther Clin Pract, 2015;21(1):52-6. DOI: 10.1016/j. ctcp.2014.12.001

27. Borges TP, Kurebayashi LFS, Silva MJP. Occupational low back pain in nursing workers: massage versus pain. Rev Esc Enferm USP. 2014;48(4):669-75. DOI: 10.1590/S0080-623420140000400014

28. Buttagat V, Eungpinichpong W, Chatchawan U, Kharmwan S. The immediate effects of traditional Thai massage on heart rate variability and stress-related parameters in patients with back pain associated with myofascial trigger points. J Bodyw Mov Ther. 2011;15(1):15-23. DOI: 10.1016/j.jbmt.2009.06.005 